\title{
CONJECTURES SUR LES DÉRIVÉES LOGARITHMIQUES DES FONCTIONS $L$ D'ARTIN AUX ENTIERS NÉGATIFS
}

\author{
Vincent Maillot and Damien Roessler
}

\begin{abstract}
RÉSUMÉ. Nous formulons plusieurs variantes d'une conjecture reliant le degré arithmétique de certains fibrés hermitiens aux valeurs prises aux entiers négatifs par la dérivée logarithmique des fonctions $L$ d'Artin, généralisant des conjectures de Colmez et Gross-Deligne et complémentant ainsi les conjectures de Beilinson pour les motifs d'Artin. Nous annonçons plusieurs résultats en direction de ces énoncés.
\end{abstract}

\begin{abstract}
We formulate several variants of a conjecture relating the arithmetic degree of certain hermitian fibre bundles with the values of the logarithmic derivative of Artin's Lfunctions at negative integers. This generalizes conjectures by Colmez and Gross-Deligne and complements Beilinson's conjectures for the Artin motives. We announce several results in the direction of these statements.
\end{abstract}

\section{Préliminaires}

On appelle variété arithmétique tout schéma régulier qui est quasi-projectif et plat sur un anneau arithmétique (au sens de Gillet-Soulé [9]). Soient $R$ un tel anneau et $\mathcal{C}$ l'ensemble des couples $(X, D)$, où $f: X \rightarrow \operatorname{Spec} R$ est une variété arithmétique sur $R$ et $D$ un diviseur de Weil à croisements normaux de $X(\mathbb{C})$. Un fibré hermitien sur $X$ à singularités logarithmiques le long de $D$ est la donnée d'un fibré cohérent $E$ sur $X$ localement libre sur $X(\mathbb{C})$ et d'une métrique hermitienne sur $\left.E_{\mathbb{C}}\right|_{X(\mathbb{C}) \backslash D}$ qui est bonne le long de $D$ au sens de [21]; on note $\bar{E}$ un tel couple. Dans tout ce qui suit $\widehat{\mathrm{CH}}^{*}(X)$ désigne l'anneau de Chow arithmétique usuel associé à $X$ (cf. [9]) et $(X, D) \mapsto \widehat{\mathrm{CH}}^{*}(X, D)$ la flèche construite par J. I. Burgos et U. Kühn de $\mathcal{C}$ vers la catégorie des groupes abéliens $\mathbb{N}$-gradués (à paraître). Cette théorie est une extension en dimension supérieure des théories de Bost (voir [3]) et Kühn (voir [18]) pour les surfaces. On obtient cette flèche en remplaçant dans une construction de Burgos (voir [5]) des formes lisses par des formes à singularités faibles le long de $D$. Elle vérifie (entre autres choses) les propriétés suivantes:

(1): le groupe $\widehat{\mathrm{CH}}^{*}(X, D) \otimes_{\mathbb{Z}} \mathbb{Q}$ est muni d'une structure naturelle d'anneau commutatif unitaire pour laquelle la $\mathbb{N}$-graduation induite est une graduation d'anneau;

Received August 16, 2001. 
(2): si $X$ est propre sur $\operatorname{Spec} R$, il existe une application «image directe » $f_{*}: \widehat{\mathrm{CH}}^{d_{X}+1}(X, D) \rightarrow \widehat{\mathrm{CH}}^{1}(R)$, où $d_{X}$ est la dimension relative de $X$ sur $R$; cette application est un homomorphisme de groupes;

(3): à tout entier $r \geqslant 0$ et tout fibré hermitien $\bar{E}$ sur $X$ à singularités logarithmiques le long de $D$, on peut associer une $r$-ième «classe de Chern 》 $\widehat{c}_{r}(\bar{E}) \in \widehat{\mathrm{CH}}^{r}(X, D)$;

(4): à tout $R$-morphisme de schémas $g: X^{\prime} \rightarrow X$ transverse à $D$, où $X^{\prime}$ est une variété arithmétique sur $R$, on peut associer un morphisme de groupes gradués «image réciproque $» g^{*}: \widehat{\mathrm{CH}}^{*}(X, D) \rightarrow \widehat{\mathrm{CH}}^{*}\left(X^{\prime}, g^{-1} D\right)$ qui est un morphisme d'anneaux lorsqu'on tensorise par $\mathbb{Q}$;

(5): l'égalité $g^{*}\left(\widehat{c}_{r}(\bar{E})\right)=\widehat{c}_{r}\left(g^{*}(\bar{E})\right)$ vaut pour tout $r \geqslant 0$;

(6): on dispose d'un morphisme «d'oubli » $\zeta: \widehat{\mathrm{CH}}^{*}(X, D) \rightarrow \mathrm{CH}^{*}(X)$ compatible à l'application image réciproque du (4) et à celle usuelle de la théorie de Chow, ainsi qu'à la graduation et à la formation des classes de Chern;

(7): on dispose pour tout entier $p \geqslant 0$ d'un morphisme $\omega: \widehat{\mathrm{CH}}^{p-1}(X, D) \rightarrow$ $Z^{p, p}(X(\mathbb{C}), D)$ et d'un complexe de groupes :

$\bigoplus_{p \geqslant 0} H_{\mathcal{D}}^{2 p-1}\left(X_{\mathbb{R}}, \mathbb{R}(p)\right) \stackrel{a}{\rightarrow} \widehat{\mathrm{CH}}^{*}(X, D) \otimes_{\mathbb{Z}} \mathbb{Q} \stackrel{(\zeta \oplus \omega)}{\rightarrow} \mathrm{CH}^{*}(X) \otimes_{\mathbb{Z}} \mathbb{Q} \oplus \bigoplus_{p \geqslant 0} Z^{p, p}(X(\mathbb{C}), D)$

où $H_{\mathcal{D}}^{2 p-1}\left(X_{\mathbb{R}}, \mathbb{R}(p)\right)$ désigne la partie invariante par conjugaison complexe de la cohomologie de Deligne réelle $H_{\mathcal{D}}^{2 p-1}(X(\mathbb{C}), \mathbb{R}(p))$ et $Z^{p, p}(X(\mathbb{C}), D)$ le $\mathbb{C}$-espace vectoriel des formes réelles de type $(p, p)$ invariantes par conjugaison et bonnes le long de $D$ au sens de [21];

(8): si l'on munit $\bigoplus_{p \geqslant 0} Z^{p, p}(X(\mathbb{C}), D)$ de la structure d'anneau définie par le produit des formes, alors le morphisme $\zeta \oplus \omega$ est un morphisme d'anneaux ; l'image par $a$ de $\bigoplus_{p \geqslant 0} H_{\mathcal{D}}^{2 p-1}\left(X_{\mathbb{R}}, \mathbb{R}(p)\right)$ est un idéal de carré nul; et si l'on note $c: \mathrm{CH}^{*}(X) \rightarrow \bigoplus_{p \geqslant 0} H_{\mathcal{D}}^{2 p}\left(X_{\mathbb{R}}, \mathbb{R}(p)\right)$ l'application cycle, alors $a(x) \cdot y=a(c(\zeta(y)) \cdot x)$, où $x \in \bigoplus_{p \geqslant 0} H_{\mathcal{D}}^{2 p-1}\left(X_{\mathbb{R}}, \mathbb{R}(p)\right), y \in \widehat{\mathrm{CH}}^{*}(X, D) \otimes_{\mathbb{Z}} \mathbb{Q}$ et le premier point - (resp. le deuxième) dans la dernière égalité désigne le produit dans l'anneau $\widehat{\mathrm{CH}}^{*}(X, D) \otimes_{\mathbb{Z}} \mathbb{Q}$ (resp. en cohomologie de Deligne); (9): enfin si $D$ est vide et $X$ projective, il existe un isomorphisme canonique de groupes gradués $\widehat{\mathrm{CH}}^{*}(X, D) \simeq \widehat{\mathrm{CH}}^{*}(X)$ qui est un isomorphisme d'anneaux lorsqu'on tensorise par $\mathbb{Q}$ et via cet isomorphisme les classes $\widehat{c}_{r}(\cdot)$ et les morphismes $f_{*}, g^{*}, \zeta, a$ et $\omega$ introduits ci-dessus coïncident avec les objets éponymes de la théorie de Gillet-Soulé (cf. [9] et [10]).

Si plus généralement on considère dans ce qui précède des cycles à coefficients dans un corps $A \subseteq \mathbb{R}$ plutôt que dans $\mathbb{Z}$, on obtient une flèche vers la catégorie des $A$-algèbres $\mathbb{N}$-graduées que l'on note alors $(X, D) \mapsto \widehat{\mathrm{CH}}^{*}(X, D)_{A}$. On veillera à ne pas confondre $\widehat{\mathrm{CH}}^{*}(X, D)_{A}$ avec $\widehat{\mathrm{CH}}^{*}(X, D) \otimes_{\mathbb{Z}} A$. 


\section{Une conjecture pour les fibrations semi-abéliennes}

Soient $p: \mathcal{A} \rightarrow B$ un schéma semi-abélien lisse de dimension relative $d_{\mathcal{A}}$ audessus de $B$ une variété arithmétique de dimension $d$ sur $R$ et $L$ un fibré en droites sur $\mathcal{A}$ ample relativement à $p$. On suppose qu'il existe un ouvert non vide $U \subseteq B$ au-dessus duquel la restriction de $\mathcal{A}$ est un schéma abélien et, par abus de langage, on note encore $U$ le plus grand ouvert ayant cette propriété. On note $S$ le complémentaire de $U$ dans $B$ dont on suppose que $D:=S(\mathbb{C})$ est un diviseur à croisements normaux dans $B(\mathbb{C})$ et l'on fait l'hypothèse que le schéma abélien dual de $\mathcal{A}_{\mid U}$ s'étend en un schéma semi-abélien sur $B$ que l'on note $\mathcal{A}^{\vee}$. Le morphisme $f_{L}: \mathcal{A}_{\mid U} \rightarrow \mathcal{A}_{\mid U}^{\vee}$ induit par $L$ s'étend en un morphisme de $\mathcal{A}$ vers $\mathcal{A}^{\vee}$ que l'on note encore $f_{L}$.

Soit $K$ une extension finie de $\mathbb{Q}$ dont on note $\mathcal{O}_{K}$ l'anneau des entiers et pour lequel on suppose que chacun des plongements d'anneaux de $\mathcal{O}_{K}$ dans $\mathbb{C}$ se factorise par $R$. On suppose donné un plongement d'anneaux $\iota: \mathcal{O}_{K} \hookrightarrow \operatorname{End}_{B}(\mathcal{A})$ où $\operatorname{End}_{B}(\mathcal{A})$ désigne l'anneau des endomorphismes du schéma en groupes $p$ : $\mathcal{A} \rightarrow B$. Par abus de langage, on écrit $\Omega_{\mathcal{A}}$ (resp. $\Omega_{\mathcal{A}} \vee$ ) pour l'image réciproque par la section unité du faisceau des différentielles de $\mathcal{A}$ (resp. $\mathcal{A}^{\vee}$ ) sur $B$. Le fibré $\Omega_{\mathcal{A}, \mathbb{C}}$ est muni de la métrique $L^{2}$ induite par $L_{\mathbb{C}}$ et $\Omega_{\mathcal{A}^{\vee}, \mathbb{C}}$ hérite de cette métrique via l'isomorphisme $f_{L, \mathbb{C}}^{*}:\left.\left.\Omega_{\mathcal{A} \vee, \mathbb{C}}\right|_{U_{\mathbb{C}}} \rightarrow \Omega_{\mathcal{A}, \mathbb{C}}\right|_{U_{\mathbb{C}}}$ induit par $f_{L}$. La métrique canonique $\operatorname{sur} \Omega_{\mathcal{A}, \mathbb{C}}$ (resp. sur $\Omega_{\mathcal{A}^{\vee}, \mathbb{C}}$ ) est bonne le long de $D$ (voir [21]). Soit $\sigma \in \operatorname{Hom}\left(\mathcal{O}_{K}, R\right)$ et $b \in \mathcal{O}_{K}$; on note $\Omega_{\mathcal{A}, \sigma, b}$ (resp. $\left.\Omega_{\mathcal{A}^{\vee}, \sigma, b}\right)$ le noyau du morphisme de faisceaux $\iota(b)-\sigma(b): \Omega_{\mathcal{A}} \rightarrow \Omega_{\mathcal{A}}$ (resp. $\left.\iota(b)-\sigma(b): \Omega_{\mathcal{A}^{\vee}} \rightarrow \Omega_{\mathcal{A}^{\vee}}\right)$ et l'on note $\Omega_{\mathcal{A}, \sigma}$ (resp. $\Omega_{\mathcal{A}^{\vee}, \sigma}$ ) le faisceau $\cap_{b \in \mathcal{O}_{K}} \Omega_{\mathcal{A}, \sigma, b}$ (resp. $\cap_{b \in \mathcal{O}_{K}} \Omega_{\mathcal{A}^{\vee}, \sigma, b}$ ). Ce dernier est un faisceau cohérent car $\mathcal{O}_{K}$ est finiment engendré comme $\mathbb{Z}$-module. On suppose que $\Omega_{\mathcal{A}, \sigma}$ (resp. $\Omega_{\mathcal{A}^{\vee}, \sigma}$ ) est localement libre sur $B$, et que les fibrés $\Omega_{\mathcal{A}, \sigma, \mathbb{C}}$ (pour tout $\sigma \in \operatorname{Hom}\left(\mathcal{O}_{K}, R\right)$ ) sont orthogonaux entre eux fibre à fibre (il en est alors de même des $\Omega_{\mathcal{A}^{\vee}, \sigma, \mathbb{C}}$ ). La métrique canonique sur $\Omega_{\mathcal{A}, \mathbb{C}}$ (resp. de $\Omega_{\mathcal{A}^{\vee}, \mathbb{C}}$ ) induit par restriction sur les $\Omega_{\mathcal{A}, \sigma, \mathbb{C}}$ (resp. les $\Omega_{\mathcal{A}^{\vee}, \sigma, \mathbb{C}}$ ) une métrique qui d'après ce qui précède est bonne le long de $D$.

Dans ces conditions, le représentant $\operatorname{ch}\left(\bar{\Omega}_{\mathcal{A}, \sigma, \mathbb{C}}\right)$ du caractère de Chern $\operatorname{ch}\left(\Omega_{\mathcal{A}, \sigma, \mathbb{C}}\right)$ déterminé par l'unique connexion hermitienne de type $(1,0)$ sur $\Omega_{\mathcal{A}, \sigma, \mathbb{C}}$ est une forme différentielle bonne le long de $D$; la forme différentielle $\operatorname{ch}\left(\bar{\Omega}_{\mathcal{A}, \sigma, \mathbb{C}}\right)$ prend place naturellement dans un complexe calculant $\bigoplus_{p \geqslant 0} H_{\mathcal{D}}^{2 p-1}$ $\left(X_{\mathbb{R}}, \mathbb{R}(p)\right)$ et détermine ainsi un élément de $\bigoplus_{p \geqslant 0} H_{\mathcal{D}}^{2 p-1}\left(X_{\mathbb{R}}, \mathbb{R}(p)\right)$ que l'on note également $\operatorname{ch}\left(\Omega_{\mathcal{A}, \sigma, \mathbb{C}}\right)$. Enfin on note $\mathcal{H}_{n}:=\sum_{j=1}^{n} 1 / j$ le $n$-ième nombre harmonique et, pour tout caractère d'Artin $\chi$ de $K$, on note $\mathbb{Q}(\chi)$ le corps engendré par l'ensemble des valeurs de $\chi$ et $\mathbb{Q}^{+}(\chi)$ le sous-corps $\mathbb{Q}(\chi) \cap \mathbb{R}$.

Conjecture 2.1. Pour tout $n \geqslant 1$, l'égalité:

$$
\frac{1}{2} \sum_{\sigma \in \operatorname{Hom}\left(\mathcal{O}_{K}, R\right)} \widehat{\operatorname{ch}}^{[n]}\left(\bar{\Omega}_{\mathcal{A}, \sigma} \oplus \bar{\Omega}_{\mathcal{A}^{\vee}, \sigma}^{\vee} \chi(\sigma)\right.
$$




$$
=-a\left(\left(\frac{L^{\prime}(\chi, 1-n)}{L(\chi, 1-n)}+\frac{1}{2} \mathcal{H}_{n-1}-\frac{c_{\chi} \log (2)}{1-2^{-n}}\right) \sum_{\sigma \in \operatorname{Hom}\left(\mathcal{O}_{K}, R\right)} \operatorname{ch}^{[n-1]}\left(\Omega_{\mathcal{A}, \sigma, \mathbb{C}}\right)\right) \chi(\sigma)
$$

vaut dans $\widehat{\mathrm{CH}}^{n}(B, D)_{\mathbb{Q}^{+}(\chi)} \otimes_{\mathbb{Q}^{+}(\chi)} \mathbb{Q}(\chi)$ pour tout caractère d'Artin irréductible $\chi$ de $K$ tel que $L(\chi, 1-n) \neq 0$, sauf éventuellement lorsque $n=1$ et $\chi$ est le caractère trivial. Ici $c_{\chi}=1$ si $\chi$ est le caractère trivial et $c_{\chi}=0$ sinon.

\section{Remarques.}

1. On notera qu'aucun plongement de $K$ dans $\mathbb{C}$ n'est fixé a priori; le fait que l'égalité ci-dessus doit être vérifiée quel que soit le plongement choisi fait partie de la conjecture.

2. En appliquant le morphisme d'oubli $\zeta$ aux deux côtés de la conjecture 2.1, on obtient un énoncé d'annulation dans le groupe de Chow $\mathrm{CH}^{n}(B) \otimes \mathbb{Q}(\chi)$ qui (à la connaissance des auteurs) est également conjectural.

3. Supposons que $R$ est un ouvert d'un anneau de corps de nombres et soit Spec $\mathbb{Z}[1 / N]$ le plus grand ouvert de $\mathbb{Z}$ au-dessus duquel Spec $R$ est fini; lorsque $B$ est propre, on écrit $\widehat{\operatorname{deg}}: \widehat{\mathrm{CH}}^{d+1}(B, D)_{\mathbb{Q}^{+}(\chi)} \rightarrow \widehat{\mathrm{CH}}^{1}$ $(\operatorname{Spec} \mathbb{Z}[1 / N])_{\mathbb{Q}^{+}(\chi)}$ pour la composée des applications «image directe 》 $\widehat{\mathrm{CH}}^{d+1}(B, D)_{\mathbb{Q}^{+}(\chi)} \rightarrow \widehat{\mathrm{CH}}^{1}(R)_{\mathbb{Q}^{+}(\chi)}$ et $\widehat{\mathrm{CH}}^{1}(R)_{\mathbb{Q}^{+}(\chi)} \rightarrow \widehat{\mathrm{CH}}^{1}$ $(\operatorname{Spec} \mathbb{Z}[1 / N])_{\mathbb{Q}^{+}(\chi)}$ introduites au 1 . De l'égalité obtenue en appliquant le morphisme $\widehat{\operatorname{deg}}$ aux deux côtés de la formule conjecturée pour $n=d+1$, on déduit une égalité de nombres réels à des sommes de termes de la forme $b_{\chi} \log q$ près, où $q$ est un entier positif divisant $N$ et $b_{\chi}$ un élément de $\mathbb{Q}(\chi)$. En particulier, si $R$ est un anneau de corps de nombres, on obtient une égalité de nombres réels.

Lorsque $K$ est un corps CM et que $n=1, d_{\mathcal{A}}=[K: \mathbb{Q}] / 2$ et $S$ est vide, la conjecture 2.1 est un léger raffinement de la conjecture de Colmez [7, Conj. 0.4, p. 632] qu'il prouve lorsque $K$ est une extension abélienne de $\mathbb{Q}$ (à un facteur $\log (2)$ près). Le cas de 2.1 où $K$ est un corps quadratique imaginaire, $n=1$ et $S$ est vide implique un raffinement d'un théorème de Gross [12, Th. 3, Par. 3, p. 204]. Si 2 est inversible dans $R$, le calcul [18, Th. 6.1, p. 229] de Kühn (voir aussi celui fait indépendamment par Bost) établit la conjecture 2.1 pour $K=\mathbb{Q}, n=2$ et $d_{\mathcal{A}}=1$ et sa conjecture [20, Cor. 2.9.14] implique la conjecture 2.1 lorsque $K$ est un corps quadratique totalement réel, $n=2$ et $d_{\mathcal{A}}=2$ (voir la proposition 2.2 ). Le cas de 2.1 où $K$ est un corps quadratique imaginaire, $d_{A}=2$ et $R$ est un certain anneau arithmétique peut être déduit d'une conjecture de Kudla, Rapoport et Yang sur les dérivées de certaines séries d'Eisenstein incohérentes (voir $[17,(0.16)])$; et les cas où $K$ est un corps quadratique totalement réel, $d_{\mathcal{A}}=2$ et $n=2$ (voir la proposition 2.2 ) et où $K=\mathbb{Q}, d_{A}=2, d=3$ et $n=2,4$ permettent de préciser une conjecture de Kudla (voir [16, (6.11) et $(6.12)])$. Enfin le cas où $K=\mathbb{Q}$ et $S$ est vide est conjecturé par Köhler dans [13, Th. 3.3]. La proposition suivante donne comme application de ce qui précède 
une formule pour le degré arithmétique des fibrés de formes modulaires sur les variétés modulaires de Hilbert, munis de leur métrique de Petersson.

Proposition 2.2. Soit $\mathcal{A} \rightarrow B$ une fibration semi-abélienne lisse munie d'une action $\iota: \mathcal{O}_{K} \hookrightarrow \operatorname{End}_{B}(\mathcal{A})$ de l'anneau des entiers d'un corps de nombres totalement réel $K$ et satisfaisant aux hypothèses de la conjecture 2.1. Supposons de plus que $B$ est propre, $d_{\mathcal{A}}=[K: \mathbb{Q}]=d$ et que $R$ est un ouvert d'un anneau de corps de nombres où le degré de $f_{L}$ est inversible. Soit $\mathbb{Z}[1 / N]$ le plus grand ouvert au-dessus duquel $R$ est fini et soient $K_{A}$ le corps engendré par les valeurs de tous les caractères d'Artin (pairs) de $K$ et $K_{A}^{+}$le sous-corps $K_{A} \cap \mathbb{R}$. L'égalité :

$$
\begin{aligned}
& \widehat{\operatorname{deg}}\left(\widehat{c}_{1}^{d+1}\left(\bar{\Omega}_{\mathcal{A}}\right)\right)= \\
& -(d+1)\left(\frac{d}{3} \frac{\zeta_{\mathbb{Q}}^{\prime}(-1)}{\zeta_{\mathbb{Q}}(-1)}+\frac{2}{3} \sum_{\chi} \chi^{2}(1) \frac{L^{\prime}(\chi,-1)}{L(\chi,-1)}+\frac{d}{2}-\frac{4(d+2)}{9} \log (2)\right) \operatorname{deg}\left(\Omega_{\mathcal{A}, \mathbb{C}}\right)
\end{aligned}
$$

dans $\widehat{\mathrm{CH}}^{1}(\operatorname{Spec} \mathbb{Z}[1 / N])_{K_{A}^{+}}$est une conséquence de la conjecture 2.1 pour $n=2$ et $\chi$ parcourant les caractères d'Artin (pairs) de $K$.

La proposition suivante donne comme application de ce qui précède une formule pour le degré arithmétique des fibrés de formes modulaires sur les variétés modulaires classifiant les surfaces abéliennes à multiplication quaternionique, munis de leur métrique de Petersson.

Proposition 2.3. Soit $\mathcal{A} \rightarrow B$ une fibration semi-abélienne lisse munie d'une action $\iota: \mathcal{O}_{K} \hookrightarrow \operatorname{End}_{B}(\mathcal{A})$ d'un anneau de corps de nombres quadratique imaginaire et satisfaisant aux hypothèses de la conjecture 2.1. Supposons de plus que $B$ est propre, $d_{\mathcal{A}}=[K: \mathbb{Q}]=d+1=2$ et que $R$ est un ouvert d'un anneau de corps de nombres où le degré de $f_{L}$ est inversible. Soit $\mathbb{Z}[1 / N]$ le plus grand ouvert au-dessus duquel $R$ est fini. L'égalité:

$$
\widehat{\operatorname{deg}}\left(\widehat{c}_{1}^{2}\left(\bar{\Omega}_{\mathcal{A}}\right)\right)=-\left(4 \frac{\zeta_{\mathbb{Q}}^{\prime}(-1)}{\zeta_{\mathbb{Q}}(-1)}-\frac{16}{3} \log (2)+2\right) \operatorname{deg}\left(\Omega_{\mathcal{A}, \mathbb{C}}\right)
$$

dans $\widehat{\mathrm{CH}}^{1}(\operatorname{Spec} \mathbb{Z}[1 / N])_{\mathbb{Q}}$ est une conséquence de la conjecture 2.1 pour $n=2$ et $\chi$ le caractère trivial de $K$ et pour $n=1$ et $\chi$ l'unique caractère d'Artin non-trivial de $K$.

A la fin d'une nouvelle version de [13], Köhler conjecture une formule pour le degré arithmétique des fibrés de formes modulaires sur les variétés modulaires de Siegel. Cette formule est une conséquence de la conjecture 2.1, pour $n$ parcourant les entiers pairs positifs et $\chi$ le caractère trivial.

Indèpendamment de ce qui précède, J. Kramer a conjecturé (conversations avec le second auteur) que le degré arithmétique du fibré des formes modulaires sur une variété de Shimura est donné par une combinaison linéaire rationnelle de 
dérivées logarithmiques de fonctions $\zeta$ de corps de nombres évaluées en des entiers négatifs. Les deux propositions prècèdentes sont compatibles avec une telle conjecture.

\section{Une conjecture pour les motifs relatifs}

Soient $Y$ une variété arithmétique lisse sur $R$ un anneau arithmétique de Dedekind et $\mathcal{V}$ un motif relatif sur $Y$ à coefficients dans $\mathbb{Z}\left[\frac{1}{m}\right]$ (avec $m$ un entier positif) et effectif pour l'équivalence rationnelle. Le motif $\mathcal{V}$ consiste par définition en un couple formé d'une fibration projective et lisse $f: X \rightarrow Y$ et d'un idempotent pour la composition des correspondances dans $\mathrm{CH}^{*}\left(X \times_{Y} X, \mathbb{Z}\left[\frac{1}{m}\right]\right)$; nous renvoyons à [8] pour la définition générale de la catégorie des motifs relatifs sur $Y$. On se donne sur $X$ un fibré en droites $L$ ample relativement à $f$. Nous écrirons $H_{\mathrm{Dlb}}(\mathcal{V})$ pour désigner la cohomologie de Dolbeault de $\mathcal{V}$ (on rappelle que si l'idempotent associé à $\mathcal{V}$ est l'identité, $H_{\mathrm{Dlb}}(\mathcal{V})$ est le faisceau $\left.\oplus_{k \geq 0} \oplus_{p+q=k} R^{q} f_{*} \Lambda^{p} \Omega(f)\right)$; c'est un faisceau cohérent $\mathbb{N}$-gradué sur $Y$. On suppose que le faisceau $H_{\mathrm{Dlb}}(\mathcal{V})_{\mathbb{C}}$ est localement libre; il est alors muni de la métrique $L^{2}$ induite par $c_{1}\left(L_{\mathbb{C}}\right) \in H_{\mathrm{Dlb}}^{2}(X / Y)_{\mathbb{C}}$.

Soit $P(T)$ un polynôme unitaire à coefficients dans $\mathbb{Z}\left[\frac{1}{m}\right]$ et irréductible sur $\mathbb{Q}$. On pose $\mathcal{O}:=\mathbb{Z}\left[\frac{1}{m}\right][T] /(P(T))$ et l'on note $K$ le corps des fractions de $\mathcal{O}$ (c'est une extension finie de $\mathbb{Q}$ ) et l'on suppose que chacun des plongements d'anneaux de $\mathcal{O}$ dans $\mathbb{C}$ se factorise par $R$. On suppose donné un morphisme d'anneaux $\iota: \mathbb{Z}\left[\frac{1}{m}\right][T] \rightarrow \operatorname{End}(\mathcal{V})$ dont le noyau est contenu dans l'idéal $(P(T))$. Pour tout $\sigma \in \operatorname{Hom}(\mathcal{O}, R)$ on note $H_{\mathrm{Dlb}}(\mathcal{V})_{\sigma}$ le noyau du morphisme de faisceaux $\iota(T)-$ $\sigma(T): H_{\mathrm{Dlb}}(\mathcal{V}) \rightarrow H_{\mathrm{Dlb}}(\mathcal{V})$ (qui est cohérent); on suppose que $H_{\mathrm{Dlb}}(\mathcal{V})_{\sigma}$ est localement libre et l'on munit $H_{\mathrm{Dlb}}(\mathcal{V})_{\sigma, \mathbb{C}}$ de la métrique induite par restriction de celle de $H_{\mathrm{Dlb}}(\mathcal{V})_{\mathbb{C}}$. On suppose de plus que les fibrés $H_{\mathrm{Dlb}}(\mathcal{V})_{\sigma, \mathbb{C}}$ (pour tout $\sigma \in \operatorname{Hom}(\mathcal{O}, R))$ sont orthogonaux entre eux fibre à fibre. Enfin on note $r$ le produit de tous les nombres premiers $q$ au-dessus desquels il existe un idéal premier $\mathcal{Q}$ de $\mathcal{O}_{K}$ tel que $\mathcal{O}_{\mathcal{Q}}$ ne coïncide pas avec $\mathcal{O}_{K, \mathcal{Q}}$.

Conjecture 3.1. Pour tout $n \geqslant 1$ et $k \geqslant 0$, l'égalité:

$$
\begin{gathered}
\frac{1}{2} \sum_{\sigma \in \operatorname{Hom}(\mathcal{O}, R)}{\widehat{\mathrm{ch}^{[n]}}}^{\left[H_{\mathrm{Dlb}}^{k}(\overline{\mathcal{V}})_{\sigma}\right) \chi(\sigma)}=-\sum_{\sigma \in \operatorname{Hom}(\mathcal{O}, R)} a\left(\left(\frac{L^{\prime}(\chi, 1-n)}{L(\chi, 1-n)}+\frac{1}{2} \mathcal{H}_{n-1}-\right.\right. \\
\left.\frac{c_{\chi} \log (2)}{\left.1-2^{-n}\right)} \sum_{p+q=k} p \cdot \operatorname{ch}^{[n-1]}\left(H_{\mathrm{Dlb}}^{p, q}(\mathcal{V})_{\sigma, \mathbb{C}}\right)\right) \chi(\sigma)
\end{gathered}
$$

vaut dans $\widehat{\mathrm{CH}}^{n}\left(Y\left[\frac{1}{m r}\right]\right)_{\mathbb{Q}^{+}(\chi)} \otimes_{\mathbb{Q}^{+}(\chi)} \mathbb{Q}(\chi)$ pour tout $k \geqslant 0$ et tout caractère d'Artin irréductible $\chi$ de $K$ tel que $L(\chi, 1-n) \neq 0$, sauf éventuellement lorsque $n=1$ et $\chi$ est le caractère trivial. Ici $c_{\chi}=1$ si $\chi$ est le caractère trivial et $c_{\chi}=0$ sinon. 


\section{Remarques.}

1. La conjecture 3.1 implique la conjecture 2.1 lorsque $Y=\operatorname{Spec} R$ et que $R$ est un corps.

2. En appliquant le morphisme d'oubli $\zeta$ aux deux côtés de la conjecture 3.1,

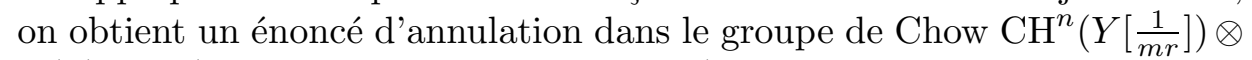
$\mathbb{Q}(\chi)$ qui (à la connaissance des auteurs) est également conjectural. On peut interpréter cet énoncé comme une version relative de la formule des traces de Lefschetz pour la cohomologie singulière à coefficients dans $\mathbb{C}$ (voir la dernière section pour plus de détails).

3. Lorsque $Y=\operatorname{Spec} \overline{\mathbb{Q}}$, la conjecture 3.1 est une extension non-abélienne d'une variation de la conjecture «des périodes 》 de Gross-Deligne [12, p. 205].

On remarquera également qu'au vu de la conjecture 2.1, on souhaiterait étendre la conjecture 3.1 à des motifs associés à des fibrations à singularités semistables ou, en d'autres termes, à une certaine sous-catégorie de la «catégorie des faisceaux en motifs mixtes $\gg$.

La conjecture suivante est une version affaiblie de la conjecture 3.1 dont elle est une conséquence immédiate:

Conjecture 3.2. Pour tout $n \geqslant 1$, l'égalité:

$$
\begin{aligned}
\frac{1}{2} \sum_{k \geqslant 0}(-1)^{k} & \sum_{\sigma \in \operatorname{Hom}(\mathcal{O}, R)} \widehat{c h}^{[n]}\left(H_{\mathrm{Dlb}}^{k}(\overline{\mathcal{V}})_{\sigma}\right) \chi(\sigma) \\
= & -\sum_{\sigma \in \operatorname{Hom}(\mathcal{O}, R)} a\left(\sum _ { k \geqslant 0 } ( - 1 ) ^ { k } \left(\frac{L^{\prime}(\chi, 1-n)}{L(\chi, 1-n)}+\frac{1}{2} \mathcal{H}_{n-1}\right.\right. \\
& \left.\quad-\frac{c_{\chi} \log (2)}{\left.1-2^{-n}\right)} \sum_{p+q=k} p \cdot \operatorname{ch}^{[n-1]}\left(H_{\mathrm{Dlb}}^{p, q}(\mathcal{V})_{\sigma, \mathbb{C}}\right)\right) \chi(\sigma)
\end{aligned}
$$

vaut dans $\widehat{\mathrm{CH}}^{n}\left(Y\left[\frac{1}{m r}\right]\right)_{\mathbb{Q}^{+}(\chi)} \otimes_{\mathbb{Q}^{+}(\chi)} \mathbb{Q}(\chi)$ pour tout caractère d'Artin irréductible $\chi$ de $K$ tel que $L(\chi, 1-n) \neq 0$, sauf éventuellement lorsque $n=1$ et $\chi$ est le caractère trivial. Ici $c_{\chi}=1$ si $\chi$ est le caractère trivial et $c_{\chi}=0$ sinon.

\section{Résultats}

Les hypothèses et les notations utilisées ci-dessous sont celles du paragraphe précédent. Les démonstrations des résultats annoncés ici seront publiées ultérieurement.

Dans tout ce qui suit, si $\chi$ est un caractère d'Artin et $H$ un corps de nombres contenant $\mathbb{Q}(\chi)$, nous dirons que la conjecture 2.1 (resp. 3.1, resp. 3.2) vaut pour $\chi$ après produit tensoriel par $H$ si la conjecture obtenue en remplaçant $\mathbb{Q}(\chi)$ par $H$ dans l'énoncé correspondant est vérifiée. De plus, pour tout entier positif 
$q$, nous noterons $\mathbb{Q}\left(\chi, \mu_{q}\right)$ le corps engendré par les valeurs de $\chi$ et les racines $q$-ièmes de l'unité.

Le théorème suivant relie la conjecture 3.2 à une formule de Lefschetz relative conjecturée dans [15, Appendix] et maintenant démontrée grâce à [15] et [2] .

Théorème 4.1. Supposons que $\mathcal{V}$ est le motif relatif associé à une fibration lisse $f: X \rightarrow Y$, que $P(T)$ est le q-ième polynôme cyclotomique et qu'il existe une racine primitive q-ième de l'unité dans $K=\mathbb{Q}\left(\mu_{q}\right)$ dont l'image par ı est représentée par un automorphisme d'ordre $q$ de la fibration $f$. Supposons de plus que $q$ est inversible dans $R$. La conjecture [15, Appendix] implique que la conjecture 3.2 vaut pour tout $\chi$ caractère de Dirichlet primitif modulo $q$ après produit tensoriel par $\mathbb{Q}\left(\chi, \mu_{q}\right)$.

Esquisse de la preuve. On montre cette implication en appliquant tout d'abord la conjecture [15, Appendix] au complexe de Dolbeault de $f$ et en utilisant le fait que la torsion analytique relative équivariante de ce complexe s'annule (voir [1]). On calcule ensuite la transformée de Fourier (pour l'action de $\operatorname{Gal}(K / \mathbb{Q})$ ) de la formule obtenue, puis en utilisant la formule des traces relatives à valeurs dans la cohomologie, on exprime la partie dépendant des points fixes en termes de traces sur la cohomologie de Dolbeault de $f$. Un argument combinatoire permet alors de conclure.

Lorsque $n=1$, comme consèquence immèdiate du théorème 4.1, ou plus simplement grâce à un cas particulier de la formule de Lefschetz conjecturale [15, Appendix] démontré dans [14], on peut affirmer que:

Corollaire 4.2. Sous les hypothèses du théorème 4.1, la conjecture 3.2 vaut après produit tensoriel par $\mathbb{Q}\left(\chi, \mu_{q}\right)$ lorsque $n=1$.

Remarque. Cet ènoncè établit en particulier une variante de la conjecture « des périodes » de Gross-Deligne pour une certaine classe de structures de Hodge CM découpées dans la cohomologie de variétés sur $\overline{\mathbb{Q}}$.

Théorème 4.3. La conjecture [15, Appendix] implique que la conjecture 2.1 vaut, après produit tensoriel par $\mathbb{Q}\left(\chi, \mu_{f_{K}}\right)$, pour tout $n \geqslant 1$ lorsque $S$ est vide et $K$ est une extension abélienne de $\mathbb{Q}$ dont le conducteur $f_{K}$ est inversible dans $R$.

Esquisse de la preuve. On utilise la théorie du corps de classes pour se ramener à $K=\mathbb{Q}\left(\mu_{f_{K}}\right)$ puis l'on montre que l'on peut déduire ce cas d'une variation de 4.1 .

Lorsque $n=1$, comme conséquence immédiate du théorème 4.3, ou plus simplement grce au cas particulier de [15, Appendix] démontré dans [14], on peut affirmer que:

Corollaire 4.4. La conjecture 2.1 est vraie après produit tensoriel par $\mathbb{Q}\left(\chi, \mu_{f_{K}}\right)$ lorsque $n=1, S$ est vide et $K$ est une extension abélienne de $\mathbb{Q}$ dont le conducteur $f_{K}$ est inversible dans $R$. 


\section{Remerciements}

Nous remercions chaleureusement J.-I. Burgos et U. Kühn pour avoir bien voulu nous expliquer leur théorie de l'intersection arithmétique avec singularités logarithmiques (voir aussi [4] et [19]). Leurs commentaires ainsi que ceux de J. Bellaïche, S. Bloch, P. Colmez, D. Harari et C. Soulé nous ont été fort utiles. Nos remerciements vont également à K. Köhler pour nous avoir communiqué [13] et pour avoir attiré notre attention sur un point de calcul qui nous avait échappé.

\section{Références}

[1] J.-M. Bismut, Holomorphic and de Rham torsions, à paraître.

[2] J.-M. Bismut, X. Ma, Holomorphic immersions and equivariant torsion forms, à paraître.

[3] J.-B. Bost, Potential theory and Lefschetz theorems for arithmetic surfaces, Ann. Sci. École Norm. Sup. (4) 32 (1999), 241-312.

[4] J.-I. Burgos, lettre aux auteurs (mars 2001).

[5] _ Arithmetic Chow rings and Deligne-Beilinson cohomology, J. Algebraic Geom. 6 (1997), 335-377.

[6] J.-I. Burgos, S. Wang, Higher Bott-Chern forms and Beilinson's regulator, Invent. Math. 132 (1998), 261-305.

[7] P. Colmez, Périodes des variétés abéliennes à multiplication complexe, Ann. of Math. (2) 138 (1993), 625-683.

[8] C. Deninger, J. Murre, Motivic decompositions of abelian schemes and the Fourier transform, J. Reine Angew. Math. 422 (1991), 201-219.

[9] H. Gillet, C. Soulé, Arithmetic intersection theory, Inst. Hautes Études Sci. Publ. Math. 72 (1990), 93-174 (1991).

[10] Characteristic classes for algebraic vector bundles with hermitian metrics. I, II. Ann. of Math. (2) 131 (1990), 163-203, 205-238.

[11] P. Griffiths, L. Tu, Variation of Hodge structure, Topics in transcendental algebraic geometry (Princeton, 1981/1982), 63-74, Ann. of Math. Stud. 106, Princeton Univ. Press, Princeton, NJ, 1984.

[12] B. H. Gross, On the periods of abelian integrals and a formula of Chowla and Selberg, Invent. Math. 45 (1978), 193-211.

[13] K. Köhler, A Hirzebruch proportionality principle in Arakelov geometry, prépublication de l'Institut de mathématiques de Jussieu no. 284 (avril 2001).

[14] K. Köhler, D. Roessler, A fixed point formula of Lefschetz type in Arakelov geometry. I. Statement and proof, Invent. Math. 145 (2001), 333-396.

[15] _ A fixed point formula of Lefschetz type in Arakelov geometry. II. A residue formula, Ann. Inst. Fourier (Grenoble) 52 (2002), 81-103.

[16] S. Kudla, Integrals of Borcherds forms, prépublication.

[17] S. Kudla, M. Rapoport, T. Yang, Derivatives of Eisenstein series and Faltings heights, prépublication.

[18] U. Kühn, Generalized arithmetic intersection numbers, J. Reine Angew. Math. 534 (2001), 209-236.

[19] , lettre aux auteurs (avril 2001).

[20] — Über die arithmetischen Selbstschnittzahlen zu Modulkurven und Hilbertschen Modulflächen, dissertation, Hu-Berlin (1999).

[21] D. Mumford, Hirzebruch's proportionality theorem in the noncompact case, Invent. Math. 42 (1977), 239-272. 
Institut de Mathématiques de Jussieu, Université Paris 7 Denis Diderot, Case Postale 7012, 2 place Jussieu, F-75251 Paris Cedex 05, France.

E-mail address: vmaillot@math.jussieu.fr

E-mail address: roessler@math.jussieu.fr 